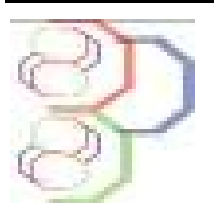

Journal of Applied Biosciences 69:5449 - 5455

\title{
Évaluation de la contamination parasitologique des eaux usées domestiques collectées et traitées à la station d'épuration de Cambérène (Dakar)
}

\author{
AKPO Y ${ }^{1 *}$, SAWADOGO G.J ${ }^{2}$, DEGNON R.G ${ }^{3}$ \\ ${ }^{1}$ Université de Parakou, Faculté d'Agronomie, Département de Production et Santé Animales, BP 123 Parakou, Bénin. \\ ${ }^{2}$ Ecole Inter-états des Sciences et Médecine Vétérinaires, BP 5077, Dakar-Fann, Sénégal. \\ ${ }^{3}$ Université d'Abomey-Calavi, Département de Génie de Technologie Alimentaire, 01 BP 2009 Cotonou, Bénin. \\ *Adresse pour correspondance Tél : (00229) 95533374 ; E-mail : adjasbj@yahoo.fr \\ Original submitted in on $20^{\text {th }}$ May 2013 Published online at www.m.elewa.org on 30 th September 2013. \\ https://dx.doi.org/10.4314/jab.v69i0.95070
}

\section{RESUME}

Objectif: Cette étude a été entreprise afin d'évaluer la contamination parasitologique des eaux usées domestiques collectées et épurées à la station à boue activée de Cambérène. Elle a permis d'une part d'identifier les œufs de différents helminthes et d'autre part de décrire la variation de la concentration temporelle de la charge parasitaire de ces eaux usées.

Méthodologie et Résultats : Les examens coprologiques effectués sur des échantillons ponctuels et séquentiels d'eaux usées avaient montré une forte charge parasitaire. La concentration moyenne en œufs d'helminthes a été de 180 œufs/l. L'évolution de la charge parasitaire au cours du temps indiquait la quasi-présence des œufs d'helminthes dans les eaux usées brutes à des concentrations variables. Par ailleurs, ces charges parasitaires enregistrées sont étroitement liées aux périodes d'activités humaines. Par contre, dans les eaux clarifiées, aucun œuf d'helminthe n'a été observé.

Conclusion et application : La charge élevée de ces eaux usées brutes en agents pathogènes interdit leur usage en agriculture contrairement aux pratiques couramment observées sur le terrain. L'abandon de cette pratique par les agriculteurs va contribuer à réduire les risques de contamination parasitaire chez l'homme. Mots clés : Eaux usées, parasitologie, helminthes, boue activée, Dakar.

Evaluation of parasitological contamination of domestic wastewater collected and treated at the wastewater treatment plant Cambérène (Dakar)

ABSTRACT

Objectives: This study was undertaken to assess the parasitological contamination of wastewaters collected and purified at the wastewater treatment plant of Cambérène. It enabled one hand to identification of the different helminth eggs and on the other hand to describe the variation of the temporal concentration of the parasite load of these wastewaters.

Methodology and Results: The coprological analysis carried out on punctual and serial samples of wastewaters showed a high parasite load. The average concentration in helminth eggs was 180 eggs/l. The evolution of parasite load in the course of time showed the presence of almost all helminth eggs in raw wastewaters at 
different concentrations. These recorded parasitic charges were closely linked to periods of human activities. In cleared up water, no helminth egg were noticed.

Conclusions and application: The high load of helminths in these raw wastewaters restricts its use in agriculture. The renunciation of wastewater re-use in agriculture will contribute to reduce human parasitological contamination.

Key words: waste waters, parasitology, helminth, active mud, Dakar.

\section{INTRODUCTION}

L'eau, source de vie est indispensable pour tout être vivant. L'importance de cette richesse naturelle est d'autant plus remarquable dans les pays à climat aride ou semi-aride qui souffrent d'un déficit hydrique et d'une irrégularité des apports d'eau dans le temps et dans l'espace. Après son utilisation, l'eau se charge en divers éléments modifiant ses caractéristiques physiques, chimiques et biologiques. Ainsi, l'eau initialement claire et potable se transforme en une eau grisâtre et usée (Niang, 1995). Les eaux usées sont des eaux résiduaires émanant d'une collectivité donnée et qui ont été préalablement utilisées pour des usages domestiques, agricoles, industriels. (Nsom Zamo, 2003). A l'heure actuelle, parmi les problèmes d'environnement les plus préoccupants, figure en bonne place le problème de l'assainissement. En effet, l'activité humaine entraîne la production d'eaux usées déchargées quotidiennement dans la nature. $\mathrm{Ce}$ rejet d'eaux usées brutes dans les milieux naturels est une pratique courante dans la plupart des pays en développement. Cette situation est accentuée dans les centres urbains à cause de la démographie galopante qui les caractérise. C'est le cas de Dakar, la capitale du Sénégal qui, en raison de l'exode rural et de la croissance naturelle rejette environ $200000 \mathrm{~m}^{3}$ d'eaux usées par jour, soit le double de la quantité déversée il y a moins de 15 ans (Sylla, 2004). Mises en contact direct avec le milieu récepteur, les eaux usées brutes engendrent des effets néfastes notamment : une nuisance liée à la stagnation de ces eaux et au dégagement d'odeurs nauséabondes; une perturbation de

\section{MATERIEL ET METHODES}

Cadre d'étude: Ce travail a été mené à la station d'épuration de Cambérène située dans la région de Dakar. Cette région urbanisée à $97 \%$ est la plus développée du Sénégal. Elle concentre l'essentiel des l'équilibre écologique du milieu naturel, surtout par la pollution des eaux de surface et des nappes phréatiques et un risque sanitaire par contamination microbiologique ou parasitaire (Diop, 1994 ; Khallaayoune, 2005 ; Sawadogo et al., 2005).

Pour atténuer ces impacts négatifs des eaux usées brutes sur l'environnement et la santé, il s'avère nécessaire de mettre en place des méthodes d'épuration des eaux résiduaires avant leur rejet ou leur réutilisation éventuelle à des fins agricoles. Aussi, divers micro-organismes pathogènes provenant essentiellement des matières fécales peuvent être rencontrés dans ces eaux usées brutes. Parmi eux se trouvent les helminthes. Les œufs d'helminthes pathogènes pour l'homme et mis en évidence dans les eaux usées appartiennent à différents groupes taxonomiques dont:

- les nématodes : Ascaris sp, Toxocara sp, Trichuris sp, Ankylostoma duodenal ;

- les cestodes: Taenia saginata, Taenia solium, Hymenolepis sp ;

- les trématodes: Fasciola sp, Schistosoma sp (Bouhoum, 1987, Asmama, 1996).

L'objectif général de cette étude est d'évaluer le niveau de la contamination parasitologique des eaux usées domestiques collectées et traitées à la station d'épuration de Cambérène à Dakar.

De façon spécifique, il s'agit :

- d'identifier les œufs d'helminthes présents dans ces eaux usées et ;

- de décrire la variation de la concentration temporelle de la charge parasitaire dans ces eaux usées.

activités industrielles et commerciales du pays. La région de Dakar héberge $54 \%$ de la population urbaine, ce qui fait d'elle une grande productrice d'eaux usées. L'implantation de la station à boue activée de Cambérène 
permet de traiter près de $10.000 \mathrm{~m}^{3}$ d'eaux usées par jour. Les eaux résiduaires qui alimentent cette station proviennent surtout des quartiers tels que Parcelles assainies, Patte d'Oie, Pikine, Almadies, Maristes, Yoff, Grand Yoff, nord et ouest Foire, Liberté VI et Guédiawaye. Le drainage de ces eaux se fait par gravitation ou par pompage à travers des canalisations. Matériel : Le matériel utilisé pour la réalisation de cette étude était composé :

- de flacons et éprouvettes de 2 litres ;

- des réactifs tels que le saccharose, le phénol, le formol, le sodium dodécyl sulfate $10 \%$;

- d'un microscope photonique de marque CETI ;

d'eaux usées ;

- d'une solution de SCHEATER constituée :

${ }^{*}$ de saccharose.............................500 g

* de phénol...................................6,5 g

* d'eau distillée..............................320 ml.

Méthodes de travail : Les prélèvements d'eaux usées brutes ont été effectués juste à l'entrée de la station d'épuration de Cambérène au niveau du bassin de convergence des différentes canalisations; quant aux eaux épurées, elles étaient prélevées dans le clarificateur situé à la fin de la filière de traitement des eaux usées. Les eaux prélevées ont été conservées par ajout du formol (2 $\mathrm{ml} / / \mathrm{d}$ 'eau) et acheminées au laboratoire.

Echantillonnage des eaux usées: Des échantillonnages séquentiels et ponctuels d'eaux usées ont été faits.

Échantillonnage ponctuel: Trois prélèvements de 2 litres chacun ont été effectués au cours de la journée à $10 \mathrm{~h}$, à $14 \mathrm{~h}$ et à $20 \mathrm{~h}$. Ces trois prélèvements de la journée étaient ensuite mélangés. De ce mélange, un échantillon composite de 2 litres a été prélevé pour analyse. Ces prélèvements étaient répétés 3 fois à raison d'un prélèvement par mois.

Échantillonnage séquentiel : II permet d'apprécier les variations journalières et au cours de la semaine.

\section{RESULTATS}

Eaux brutes: Les analyses effectuées ont permis d'identifier des œufs d'helminthes appartenant à la classe des nématodes et à celle des cestodes. Les genres identifiés étaient : Ascaris sp ; Trichuris sp, Strongyloïdes
Variation journalière : Douze prélèvements de 2 litres chacun à $2 \mathrm{~h}$ d'intervalle en $24 \mathrm{~h}$ étaient effectués pour apprécier la variation temporelle journalière. Ces prélèvements ont été répétés 3 fois à raison d'un prélèvement tous les 15 jours.

Variation au cours de la semaine : Sept prélèvements de 2 litres chacun ont été effectués à $11 \mathrm{~h}$ du lundi au dimanche afin d'apprécier la variation temporelle au cours de la semaine, les prélèvements ainsi réalisés étaient répétés 3 fois à raison d'un prélèvement par mois.

Analyses parasitologiques: Les analyses parasitologiques sur les eaux usées brutes comme sur celles épurées s'étaient déroulées au laboratoire de la station d'épuration de Cambérène à Dakar. Au total, 63 prélèvements d'eaux usées ont été analysés dont 60 prélèvements d'eaux usées brutes et 3 prélèvements d'eaux usées clarifiées. La méthode utilisée pour ces analyses est celle dite d'ARTHER- FITZGERALD-FOX, décrite dans le manuel de l'OMS (Ayres et Mara, 1997) sur l'analyse des eaux résiduaires en vue de leur recyclage en agriculture. II s'agit d'une technique de purification fondée sur la différence de densité entre le liquide de dilution et les éléments parasitaires.

Dénombrement des œufs : La cellule de MAC MASTER a été utilisée pour la quantification des œufs. C'est une lame constituée de deux chambres; chacune d'elles porte un réseau carré de $10 \mathrm{~mm}$ de côté et une profondeur de $1,5 \mathrm{~mm}$; chaque réseau est divisé en 6 rectangles égaux pour faciliter la lecture. Le volume de lecture est de $0,15 \mathrm{ml}$ et le comptage se fait au grossissement $x 100$.

Analyses statistiques : La procédure «Proc freq» du logiciel SAS (Statistical Analysis System, 1991) a été utilisée pour le calcul des fréquences des helminthes identifiés. Quant aux charges parasitaires, la procédure des modèles linéaires généralisés (Proc GLM) du logiciel SAS a été utilisée pour l'analyse de variance. Les moyennes des différents lots ont été comparées par le test $t$ de Student.

sp ; Enterobius sp ; Ankylostoma sp et Hymenolepis sp. Ces différents œufs sont rencontrés dans des proportions variables comme l'indique le tableau 1. 
Akpo et al. J. Appl. Biosci. 2013. Évaluation de la contamination parasitologique des eaux usées domestiques à la station d'épuration de Cambérène (Dakar)

Tableau 1 : Fréquence des parasites identifiés

\begin{tabular}{lllllll}
\hline $\begin{array}{l}\text { Genres de } \\
\text { parasites }\end{array}$ & Ascaris & Strongyloïdes & Enterobius & Trichuris & Ankylostoma & Hymenolepis \\
\hline $\begin{array}{l}\text { Fréquence }(\%) \\
\text { Intervalle de }\end{array}$ & $60^{\mathrm{a}}$ & $25^{\mathrm{b}}$ & $6,6^{\mathrm{c}}$ & $5^{\mathrm{c}}$ & $1,7^{\mathrm{c}}$ & $1,7^{\mathrm{c}}$ \\
confiance & & 8,4 & 4,8 & 4,2 & 2,5 & 2,5 \\
\hline
\end{tabular}

$a, b, c$ : les fréquences suivies d'une lettre différente, diffèrent significativement au seuil de $5 \%$.

Le genre Ascaris a été le plus rencontré par rapport aux autres genres $(P<0,05)$, ensuite vient le genre Strongyloïdes qui était plus fréquent que les genres Enterobius, Trichuris, Ankylostoma et Hymenolepis.
Aucune différence significative n'a été observée entre ces 4 derniers genres $(P>0,05)$. L'étude de la variation journalière des œufs d'helminthes donne les résultats consignés dans la figure 1.

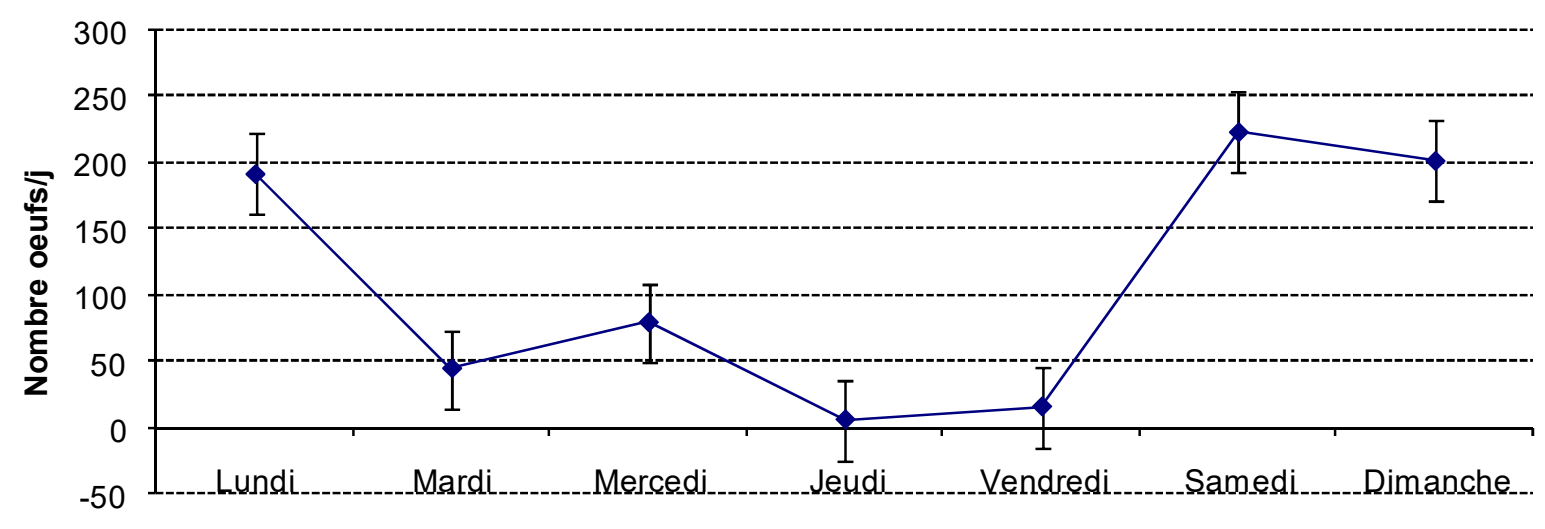

Jours de prélèvements

Figure 1 : Evolution journalière de la concentration des œufs d'helminthes

Les charges parasitaires les plus élevées $(P<0,05)$ ont été enregistrées entre $7 \mathrm{~h}$ et $12 \mathrm{~h}$ avec un pic à $11 \mathrm{~h}$. Puis la charge parasitaire avait chuté entre $13 \mathrm{~h}$ et $1 \mathrm{~h}$ du matin. Enfin, des concentrations faibles en œufs d'helminthes étaient enregistrées entre $1 \mathrm{~h}$ et $5 \mathrm{~h}$ du matin. La variation de la charge parasitaire au cours de la semaine est indiquée dans la figure 2.

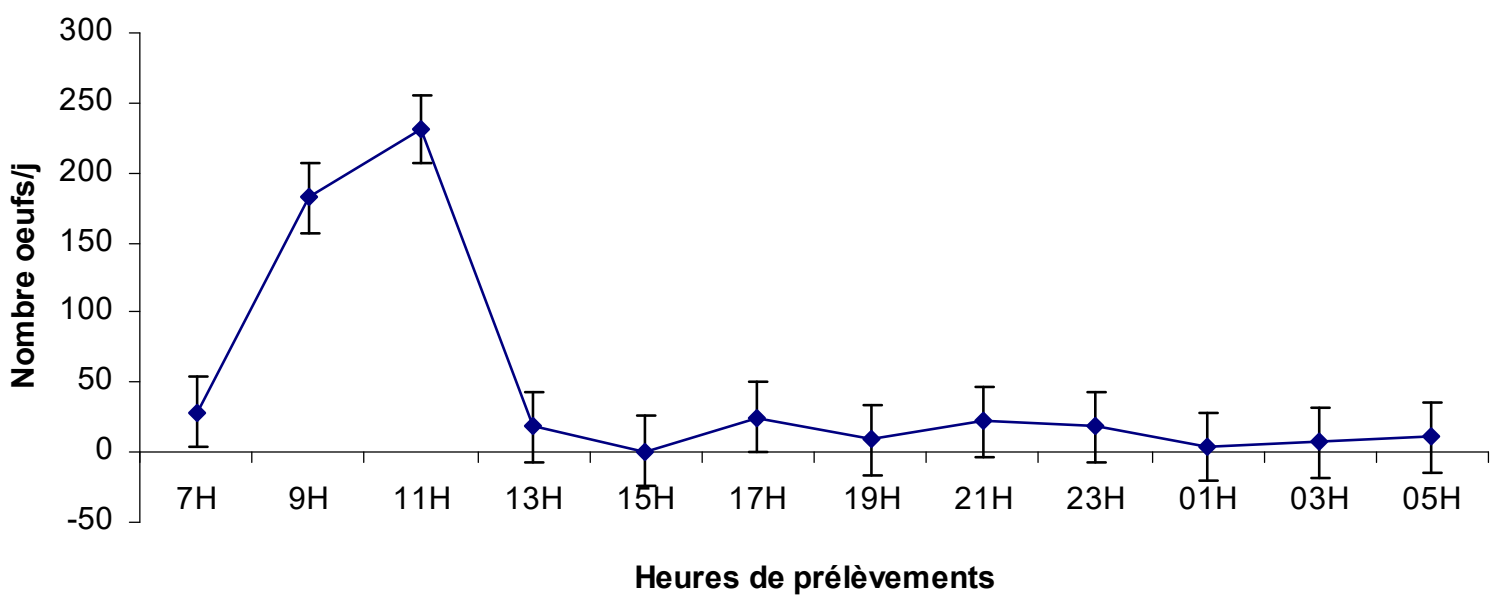

Figure 2 : Evolution de la concentration des œufs d'helminthes au cours de la semaine 
En fonction des charges parasitaires obtenues, deux périodes peuvent être distinguées dans la semaine. La première période allant du mardi au vendredi avec une concentration en œufs faible $(P<0,05)$ et la seconde période part du samedi au lundi avec des concentrations en œufs élevées $(P<0,05)$.

\section{DISCUSSION}

De nos résultats, il ressort que la concentration moyenne en œufs d'helminthes des eaux usées brutes est de 180 œufs/l. Ceci révèle que ces eaux usées sont très chargées. Ces œufs proviennent essentiellement des matières fécales de l'homme et des animaux. Ainsi, cette forte concentration en œufs traduit le taux d'infestation de la population humaine et animale des quartiers desservis par le réseau d'égout. Cette charge en œufs d'helminthes est supérieure à celles rapportées par Asmama (1996) et Karamoko (2000) sur les eaux usées au Maroc qui sont respectivement de 7,56 et 14,4 œufs /I. Cette valeur est par contre proche des concentrations trouvées par Ayres et Mara (1997) dans les eaux usées au Kenya et au Brésil qui sont respectivement de 158 et 184 œufs/l. II faut cependant signaler que la concentration en œufs d'helminthes dans les eaux usées est très variable et subordonnée aux conditions sociales, économiques, sanitaires et climatiques. Les différents genres de parasites rencontrés dans les eaux usées peuvent traduire le polyparasitisme qui sévit dans la zone concernée. Cent pour cent des échantillons composites sont positifs. La répartition des œufs d'helminthes identifiés montre une prédominance des œufs de nématodes $(98,3 \%)$, preuve de leur transmission facile et de leur pouvoir de contamination élevé. Cette prédominance des œufs de nématodes a été également signalée d'une part par Khallaayoune (1996) et d'autre part par Dssouli et al. (2001). Les œufs de cestodes par contre ne représentent que 1,7\%. Quant aux trématodes, leurs œufs ne sont pas rencontrés. L'absence des œufs de trématodes serait liée à la densité de ces œufs, ce qui traduit les performances modestes de la méthode d'ARTHER- FITZGERALD-FOX pour les œufs de cette classe. L'analyse des variations de la charge parasitaire des eaux résiduaires au cours de la journée révèle une étroite corrélation entre la concentration des œufs d'helminthes et le degré des activités humaines. Ainsi, les faibles concentrations coïncident avec les moments de repos pendant la nuit ( $1 \mathrm{~h}$ à $5 \mathrm{~h}$ ), tandis que les charges parasitaires élevées sont obtenues durant les périodes d'activités ( $7 \mathrm{~h}$ à $23 \mathrm{~h}$ ). II est à souligner également que dans la journée, les plus importantes charges parasitaires sont observées aux moments des toilettes corporelles à
Eaux épurées : Encore appelées eaux clarifiées, les résultats des analyses parasitologiques effectuées sur celles-ci ont révélé l'absence d'œuf d'helminthe. Ainsi, l'efficacité épuratoire de la méthode par boue activée de la station de Cambérène était de $100 \%$.

domicile, le matin de $7 \mathrm{~h}$ à $11 \mathrm{~h}$ et le soir entre $17 \mathrm{~h}$ et 21 h. Cette variation de la concentration des œufs d'helminthes pendant la journée permet de montrer que la charge parasitaire atteint son pic à $11 \mathrm{~h}$. II est à signaler que le drainage des eaux usées de leur site de production à la station d'épuration dure en moyenne $3 \mathrm{~h}$. Le taux d'échantillons positifs (83\%) traduit la quasiprésence d'œufs d'helminthes dans les eaux usées quelle que soit l'heure de la journée.

Au cours de la semaine, $81 \%$ des échantillons analysés se sont révélés positifs, ce qui indique une richesse des eaux usées en œufs d'helminthes tout au long de la semaine. Au regard de ces résultats, il ressort que les charges parasitaires les plus importantes sont observées en fin de semaine. Ceci pourrait être dû à la présence des familles à leur domicile en cette période et aux intenses activités ménagères.

Ces observations sur la variation journalière et au cours de la semaine sont semblables à celles faites au Maroc par Belguyti et al. (2003) qui avaient signalé que le pic de la concentration en œufs au cours de la journée est atteint à $11 \mathrm{~h}$, et les fortes charges parasitaires dans la semaine sont enregistrées en fin de semaine.

L'absence d'œuf d'helminthe dans les eaux épurées indique qu'elles peuvent être réutilisées en agriculture, car conformes aux normes de l'O.M.S, qui préconisent une concentration d'au plus 1 œuf / I. Actuellement, ces eaux épurées sont en partie utilisées pour irriguer l'espace vert du Technopôle de Dakar, le reste étant déversé dans la mer au niveau de Cambérène.

Parallèlement, les eaux usées brutes d'après les travaux de Niang (1995) sont utilisées par les maraîchers pour l'irrigation de leurs cultures. En effet, dans certains quartiers tels que Ouakam et Pikine, les maraîchers détournent une partie des eaux usées brutes vers leurs champs en greffant au collecteur principal des collecteurs secondaires. Aussi, il est à signaler que le volume moyen des eaux usées drainées quotidiennement vers la station d'épuration de Cambérène soit $15000 \mathrm{~m}^{3}$, représente environ le dixième de la production totale d'eaux usées par jour à Dakar. Ceci montre le volume limité des eaux usées sur lequel notre travail a porté. En effet, la principale station d'épuration de l'Office National de 
l'Assainissement du Sénégal à Dakar est constituée par celle de Cambérène, traduisant une insuffisance de

\section{CONCLUSION}

L'eau peut devenir une source de maladie lorsque sa qualité se dégrade. En effet, après son utilisation l'eau initialement potable se transforme en une eau grisâtre et usée. La caractérisation parasitologique effectuée sur les eaux usées domestiques collectées à la station d'épuration de Cambérène montre qu'elles sont chargées en œufs d'helminthes. Le niveau de cette charge parasitaire témoigne d'une forte infestation de la population humaine et animale de la zone concernée. Par ailleurs, ces charges parasitaires enregistrées sont étroitement liées aux périodes d'activités humaines. Ainsi au cours de la journée, la concentration la plus importante est observée autour de $11 \mathrm{~h}$ du matin, tandis que les charges les plus élevées au cours de la semaine

\section{REMERCIEMENTS}

Nous adressons nos sincères remerciements aux autorités de l'EISMV et de l'IAV Hassan II. Nous remercions également l'Agence Universitaire de la Francophonie (AUF) qui a financé notre stage au Maroc,

\section{REFERENCES BIBLIOGRAPHIQUES}

Asmama S, 1996. Traitement et réutilisation des eaux usées en agriculture (Marrakech): aspect parasitologique. Thèse de DES de 3 ème cycle : Marrakech; 122.

Ayres RM. et Mara DD, 1997. Analyse des eaux résiduaires en vue de leur recyclage en agriculture. Manuel des techniques de laboratoire en parasitologie et bactériologie. Genève : OMS.- $30 \mathrm{p}$.

Belghyti D, El Kharrim K, Bachikh J, Gabrion C, 2003. Caractérisation parasitologique des eaux usées du Lac Fouarat (Kénitra-Maroc) et évaluation du niveau épidémiologique dans une population rurale en contact avec ces eaux. Actes Inst. Agron. Vet., 21 (4) : 155-177.

Bouhoum K, 1987. Devenir des œufs d'helminthes au cours d'un traitement expérimental d'épuration par bassin de stabilisation- Marrakech. Thèse $3^{\text {ème }}$ cycle en Sciences de la vie : Marrakech ; 159.

Diop BS, 1994. Traitement des eaux usées domestiques par voie naturelle, sous climat tropical. Mémoire de DEA Environnement, ISE : Dakar ; 322.

Dssouli K, Kharboua M, Khallaayoune K, Haloui B, El Halouani $H, 2001$. Etude de la contamination l'assainissement liquide.

sont enregistrées en fin de semaine. Les œufs d'helminthes sont quasi-présents dans les eaux usées brutes avec une prédominance des œufs de nématodes. Malgré cette forte contamination parasitaire, les eaux usées brutes continuent d'être réutilisées en maraîchage dans la région de Dakar. La limitation des risques attribuables aux eaux usées passe non seulement par l'abandon des pratiques actuelles de réutilisation de ces eaux, mais aussi par le respect des règles d'hygiène en ce qui concerne les produits maraîchers consommés à l'état cru. Toutefois, la méthode par boue activée utilisée à la station de Cambérène s'avère efficace avec un abattement de $100 \%$ des œufs d'helminthes.

l'administration et le personnel de l'Office National de l'Assainissement du Sénégal (ONAS).

parasitologique des cultures irriguées par les eaux usées dans le Maroc Oriental. Actes Inst. Agron. Vet., 21 (4): 215-225.

Karamoko Y, 2000. Caractérisation parasitologique de deux rejets d'eaux usées dans l'Oued Martil (Tétouan). Mémoire de DESA Biotechnologies Toxico- génétique et santé, FST : Tétouan ; 57.

Khallaayoune K, 1996. Réutilisation des eaux usées en agriculture : risque de transmission de parasites à l'homme et à l'animal. Homme, Terre et Eaux, 26 (103) : 14-19.

Khallaayoune K, 2005. Epidémiologie des maladies parasitaires liées aux eaux usées : situation au Maroc et stratégies de prévention (4) in compte rendu Atelier- séminaire sur traitement et réutilisation des eaux usées: impact sur la santé et l'environnement du 3-6 mars. Rabat : IAV.- $14 \mathrm{p}$.

Niang S, 1995. Evacuation et traitement des eaux usées urbaines de Dakar: bilan de la situation, comportements des populations et perspectives d'avenir : premières contributions pour le choix d'un système de traitement des eaux usées urbaines de Dakar par Mosaïques Hiérarchisées 
d'Ecosystèmes Artificiels. Thèse de 3ème cycle en Sciences de l'Environnement : Dakar ; 90.

Nsom Zamo AC, 2003. Pollution par les eaux usées : Analyse parasitologique des eaux usées brutes et des cultures maraîchères, étude de la viabilité des helminthes et évaluation du risque sanitaire humain dans la région du Gharb (Kénitra). Thèse de Doctorat de Parasitologie : Kénitra ; 152.

Sawadogo GJ, Téko-agbo A, Akpo Y, 2005. Réutilisation des eaux usées en agriculture au Sénégal: impact sur la santé etl'environnement in compte rendu Atelier- séminaire sur traitement et réutilisation des eaux usées: impact sur la santé et l'environnement du 3-6 mars. Rabat : IAV.- $14 \mathrm{p}$.

Sylla C, 2004. Epuration des eaux usées des villes pour soutenir l'agriculture: une manne liquide: [Ressource électronique]- accès internet. URL.http://www.idrc.ca/fr/ev-63846-201-1DO_TOPIC.html. 